\title{
Next Generation Mobility Applications: A Wireless Cooperative Access Architecture
}

\author{
Cristian Perra, Senior Member, IEEE
}

\begin{abstract}
Accessing cellular networks for downloading large-size contents is a common task performed from modern smart phones. Nevertheless, several problems need to be addressed in order to provide an acceptable quality of experience to the final users. This paper presents a smart phone application aiming at reducing both cellular network load and file downloading time by leveraging network resource sharing. Groups of neighboring devices are formed depending on geographical position and battery consumption. WWAN and WLAN are then shared under an altruistic cooperative scenario for an increased user experience when accessing multimedia content.
\end{abstract}

Keywords - Cooperative access, Network cooperation.

\section{INTRODUCTION}

$\mathrm{C}$ ooperative download methods based on the estimation of proximity among smart phone devices and on the measure of energy consumption have shown to provide improvements in terms of reduction of cellular network load [1].

User expectations while accessing multimedia content from mobile devices are often neglected for reasons that are mainly related to the time varying nature of overlay networks. Fast spreading of smart phone devices is fostering novel applications for enabling cooperative access to multimedia resources that exploits cooperation between devices by sharing network resources [2].

The need of social elements to make egoistic users cooperate with each other in certain situations is discussed in [3] employing network coding as the enabling technology for making user cooperation more efficient.

Several research activities have analyzed and proposed client-server applications for optimizing the access to remote digital images [4]-[8].

More recent research activities are exploring the possibility of increasing the network throughput by exploiting unused network resources available in

Paper received February 24, 2014; revised April 3, 2014; accepted April 23, 2014. Date of publication July 31, 2014. The associate editor coordinating the review of this manuscript and approving it for publication was Prof. Jovan Đorđević.

This paper is a revised and expanded version of the paper presented at the 21th Telecommunications Forum TELFOR 2013.

The author gratefully acknowledges Sardinia Regional Government for the financial support (P.O.R. Sardegna F.S.E. Operational Programme of the Autonomous Region of Sardinia, European Social Fund 2007-2013 - Axis IV Human Resources, Objective 1.3, Line of Activity 1.3.1).

Cristian Perra is with the Department of Electrical and Electronic Engineering, University of Cagliari, Via Marengo, 2, 09123 Cagliari, Italy (e-mail: cperra@diee.unica.it). neighboring devices [9]. Other research activities are exploring the possibility offered by dynamic spectrum access and cognitive radio paradigm [10].

While the standard cellular system implies a direct connection between the mobile device and the base station, cooperative wireless networks set up short range links to neighboring mobile devices.

In [11] a framework for collaborative download in wireless mobile environments has been presented. The challenges and task identified are related to three aspects: group formations, where a collaborative group is formed depending on the physical location of devices, the available resources, and interests of users; the workload distribution, which addresses the identification of a designated part to be downloaded by each device; file exchange, where every device downloads a unique part of the file using WWAN technology and on completion of download each device exchanges downloaded parts using WLAN

In [12], the authors propose a mobile proximity-based content sharing application. A content sharing service on portable devices has been implemented on tablets in order to analyze the feasibility of content sharing applications.

Technologies such as network coding [13] can help in the design of wireless cooperative access architectures.

An architecture for wireless mesh networks based on network coding has been presented in [14]. The main objective is the throughput improvement exploiting network coding and the broadcast nature of the wireless channel. Nodes of a wireless mesh network encode packets from different sources in order to increase the information content of each transmission, reduce the number of transmissions, and hence increase the throughput.

A performance evaluation of network coding for cooperative wireless networks is presented in [15]. Network coding is implemented on consumer mobile devices while performance is analyzed in terms of throughput, delay and energy consumption. Moreover, a scenario of cooperative wireless networks, where the mobile device establishes short range links to neighboring mobile devices within its proximity has also been discussed.

In [16], WLAN cooperation and structured network coding are exploited for the definition of an architecture aiming at improving broadcast video quality affected by WWAN packet losses.

Recent research activities [3] are focusing on the need of social elements to make egoistic users cooperate with 
each other. While technology-cooperation can cover several scenarios like, e.g., file download from a server, or audio-video streaming, the exploitation of social network could persuade the users to collaborate. Moreover, network coding technology is exploited for making user cooperation more efficient.

In [17] a system for collaborative download has been presented. Devices exploit both WWAN and WLAN data links for increasing the effective download speed. The system makes use of: HTTP application level connection for file downloading; cost modeling that depends on WWAN charges and energy; accounting scheme for tracking credit and debits originated by the computed costs; collaboration group formed relying on a protocol enabling each device to join a group.

Cooperative video streaming on smart phones is presented in [18]. The architecture consists of a set of smart phones cooperating for downloading the same video. One of the devices acts as a coordinator of the downloading process, instructing all the devices about those segments to be downloaded. A P2P module is responsible for distributing the content over the WLAN by packet broadcasting.

The benefits of coding and cooperation over broadcasting solutions for the network and user side have been presented in [19]. It has also been discussed how the gain of user cooperation depends on the scenario and provides the largest gains where the clouds are larger.

A scenario of cooperative wireless networks, where the mobile device establishes short range links to neighboring mobile devices within its proximity has been presented in [20].

A social television system enabling mobile users to import a live or on-demand video has been proposed in [21]. Within this system, a cloud computing paradigm as infrastructure-as-a-service or as platform-as-a-service is exploited for improving the overall quality of the system and the user experience.

This paper extends the work presented in [22], presenting a smart phone application that exploits geographical position, battery consumption, and altruistic cooperation for next generation mobility applications.

The outline of this paper is as follows. Section II presents the proposed method for forming groups of neighboring smartphones. Section III presents the architecture design overview. Section IV presents the experimental results of the proposed method. Section V concludes the paper.

\section{Proposed Method}

We propose a method for forming groups of neighboring smartphones exploiting cooperative access for reducing both cellular network load and downloading time.

Each smartphone generates a UUID (universally unique identifier) for a software identification of the device, collects the GPS coordinates, IP address, and the battery level, as summarized in Table 1.

Smart phones communicate the parameters to the server $\mathrm{S}$. These parameters are processed by the server $\mathrm{S}$ in order to form cooperative groups between the smart phones having geographical distance below a given threshold and the remaining energy above a given threshold.

Fig. 1 shows the reference scenario in which it is assumed that smartphones $\mathrm{U}, \mathrm{N}_{\mathrm{i}}$, and $\mathrm{N}_{\mathrm{i}+1}$ belong to the same cooperative group.

When a user $U$ makes a request for a file, the server $\mathrm{S}$ notifies to all smart phones belonging to the same group a request for cooperation in downloading a file.

Server S splits the file into small chunks for being distributed to the cooperative group. A cost function based on the weighted sum of geographical distance and the remaining energy is employed for statistical multiplexing of the file chunks towards cooperative smart phones.

A symmetric key cryptosystem is adopted in order to ensure confidentiality of the data transferred between server and user. The data routed by the neighboring device must be protected from unauthorized access, i.e., the communication between the server and the client through the neighboring devises must be encrypted.

The point-to-point connection between the server and the user is assumed to be a trusted connection in the sense that the security aspects are addressed by the overlay network. For this reason communications between the server and the user are not encrypted, at least for the application layer defined in this paper.

During the initialization phase, before transmission of the chunks, the server generates a symmetric key that is communicated to smartphone U.

Chunks to be sent to neighboring devices $\left(\mathrm{N}_{\mathrm{i}}\right)$ are encrypted using the symmetric key. WWAN is finally enabled for local transmission of the received chunks from neighboring devices to the smartphone $U$.

With respect to the smartphone $U$, cellular network load reduction is accomplished by receiving data packets through WLAN from neighboring cooperative devices.

We implemented the proposed method in a client-server application for image browsing [4] for evaluating the performance of the proposed system. Access to image data is the use case chosen for the evaluation while the approach is easily extensible to other file types.

\section{DESIGN OVERVIEW}

As discussed in the previous section, the architecture is composed by a server application and a client application. In this section, the main functions that have been designed and implemented are summarized.

Fig. 2 shows the networking protocols involved in the proposed architecture.

The HyperText Transfer Protocol (HTTP) is the protocol used for the communication between client nodes and the server.

The TCP/IP is the protocol used for the communication between neighboring devices.

A simple protocol has been designed for implementing the communication functionalities between the nodes.

The server provides the following main functions: cooperative group formation, file partitioning, statistical multiplexing, symmetric encryption, and file transfer management. 
TABLE 1: PARAMETERS COLLECTED BY THE CLIENT AND TRANSMITTED TO THE SERVER

\begin{tabular}{cc}
\hline Parameter & Description \\
\hline UUID & Universally unique identifier \\
$(\mathrm{X}, \mathrm{Y}, \mathrm{Z})$ & Geographical coordinates \\
IP & IP address \\
$\mathrm{E}$ & Battery level and charging state \\
\hline
\end{tabular}

The client provides the following main functions: collecting parameters to be sent to the server, accepting incoming requests for cooperation, managing chunks download and transfer to the smartphone originating the request $(\mathrm{U})$.

The main tasks performed by the server for the definition of the cooperative network are as follows:

- the devices in geographical proximity are candidates

for joining the cooperative group;

- the candidate devices are sorted by decreasing energy E;

- the user device (U) which is the final destination for a given file is elected as router $\mathrm{Wi}-\mathrm{Fi}$, hence this client will enable the router Wi-Fi mode for being contacted by neighboring devices;

- the remaining (neighboring) devices are elected client $\mathrm{Wi}-\mathrm{Fi}$ and will connect to the router $\mathrm{Wi}-\mathrm{Fi}$ for forwarding incoming data packets;

- the file to be transmitted is segmented and each segment is transmitted to a different cooperating devices;

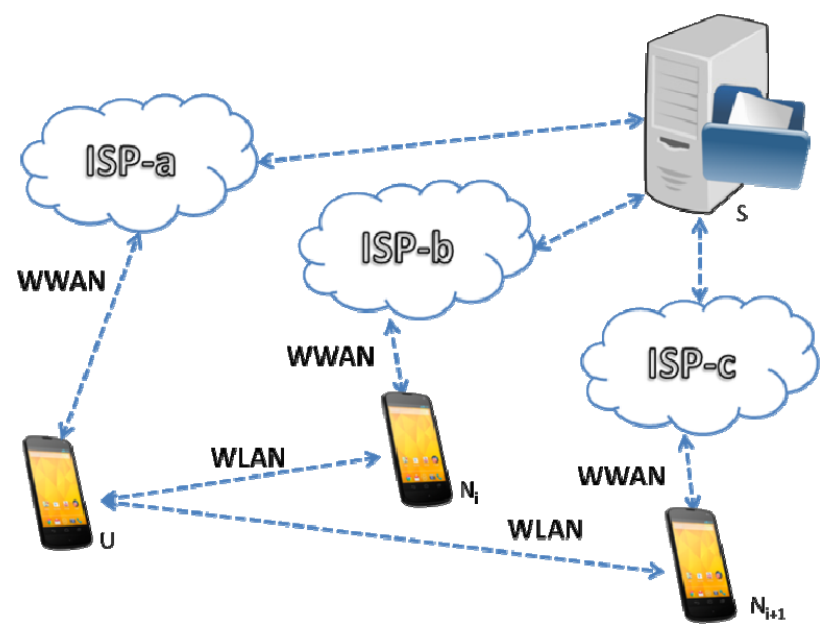

Fig. 1. Reference scenario. User (U) is requesting a file from server $(\mathrm{S})$. Neighboring devices $\left(\mathrm{N}_{\mathrm{i}}\right)$ cooperate in the download process by sharing their WWAN/WLAN connection.

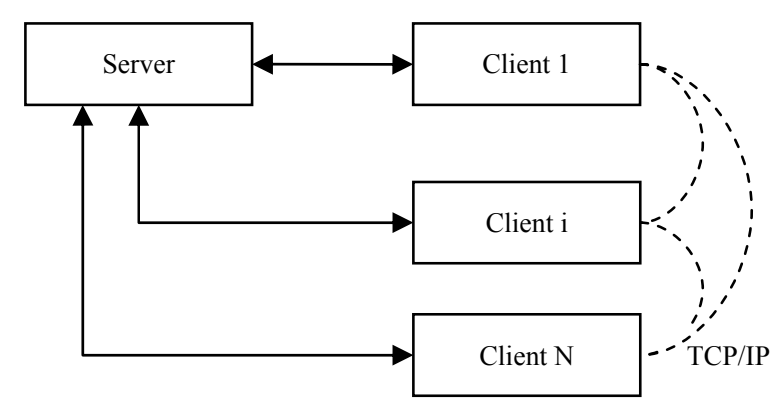

Fig. 2. Network protocols for node communications.
- a random key is generated for data encryption with the advanced encryption standard (AES), the encryption procedure is applied to the data packets sent to neighboring devices, while the random key is communicated by the server to the requesting user (U).

The main client tasks are as follows:

- to transmit parameters to the server;

- to request for a file;

- to enable the Wi-Fi router if the server notifies the possibility of collaborative download;

- to receive packets (file chunks) from the $3 \mathrm{G}$ network and from the Wi-Fi network;

- to acknowledge a received packet from both networks (3G / Wi-Fi).

- The main neighboring device actions are as follows:

- to transmit parameters to the server;

- to receive incoming packets

- to forward packets to the Wi-Fi router.

A client-server image browsing application [4] has been used as a starting point for the implementation of the proposed architecture defined in Section II.

Two additional blocks have been added: 'neighboring network topology', managing the collection of client parameters and role setting for the clients; 'client parameters', managing the collection and transfer of client parameters.

It should be pointed out that the proposed methodology is not strictly related to any particular image coding standard and the choice of [4] as a starting point was simply due to the availability of reusable modules (in particular the 'Image compositor' block) fitting some of the modules required by the proposed architecture.

Android based smart phones have been used as testing clients.

The image server has been installed on a Linux machine running the image server implementing the proposed architecture.

\section{EXPERIMENTAL RESULTS}

This section describes the experimental results of the proposed method. The testing environment is composed of a server Linux machine implementing the proposed architecture and four Android based smartphones where the client apps have been installed and operate as user and neighbors in the cooperative download process.

The reference architecture is shown in Fig. 3. The additional blocks are 'neighboring network topology', managing the collection of client parameters and role setting for the clients, and 'client parameters', managing the collection and transfer of client parameters.

The parameters considered by the test scenario have been: number of neighbors, allowed channel capacity of neighbors, image file size, number of the file requested for a single download session.

The number of neighbors examined has been between 0 (reference scenario) and 3.

For user data forwarding, we have considered the case where neighbor clients allowed for $10 \%, 20 \%, 40 \%, 60 \%$, $80 \%$, or $100 \%$ of the available channel capacity. 
Server

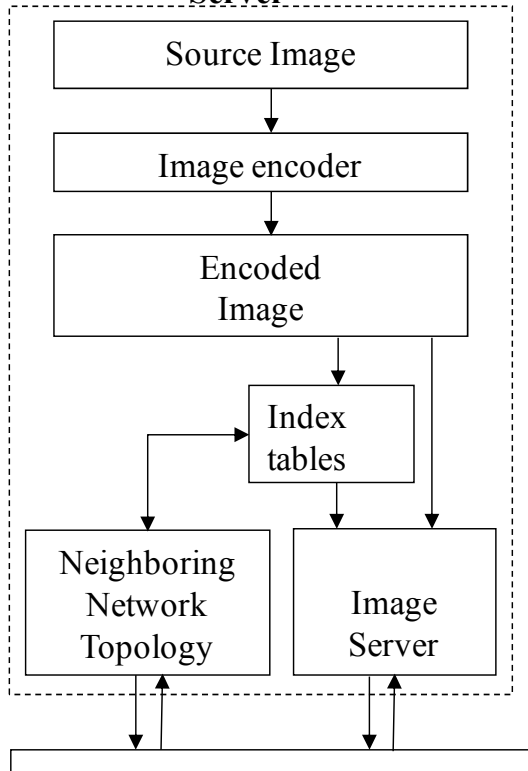

Client i

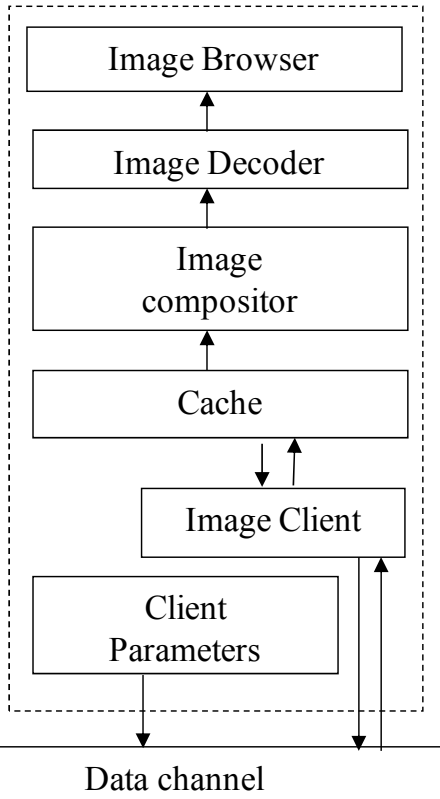

Client $\mathbf{j}$

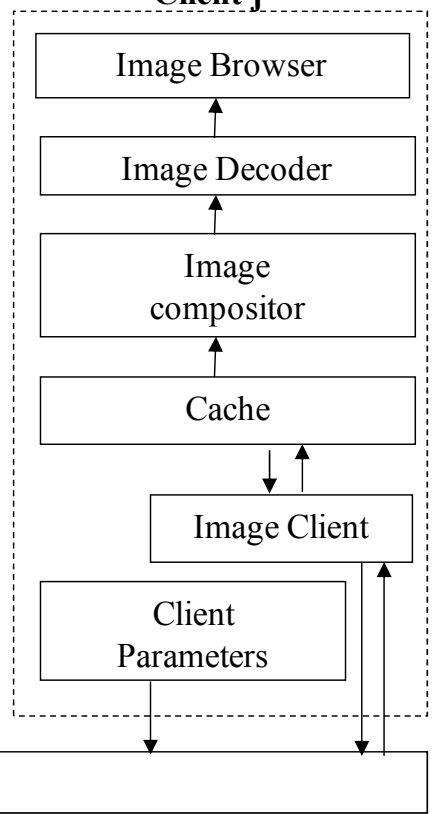

Fig. 3. Client-server architecture.

TABLE 2: EXPERIMENTAL SETUP PARAMETERS

\begin{tabular}{cc}
\hline Description & Testing parameters \\
\hline Number of neighbors & $0,1,3$ \\
\hline Data file size & 10MByte, 50MByte, 100MByte \\
\hline
\end{tabular}

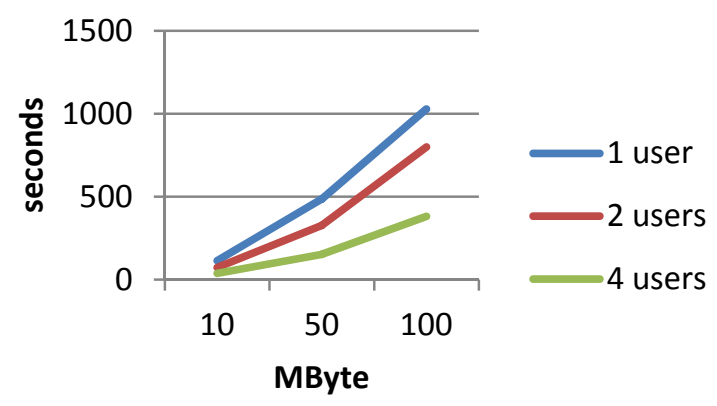

Fig. 4. Downloading time for 1 user (no cooperation), 2 users ( 1 cooperating device), 4 users ( 3 cooperating devices).

Two datasets of images have been considered, the first one with image size averaging 1 Mbytes and the second one with image size averaging 5Mbytes. Finally, for the number of continuous requested files, the considered cases have been: single file, 10 files, and 100 files.

Table 2 summarizes the parameters of the experimental setup. The reference scenario is a single smart phone with a $3 \mathrm{G}$ connection. The smartphone is downloading images directly from the image server.

The number of neighbors examined is: 0 (reference scenario), 1 , and 3 . The ' 0 neighbors' case corresponds to a direct connection between server and client. The '1 neighbors' case corresponds to a group of two users sharing their network resources. The ' 3 neighbors' case corresponds to a group of four users sharing their network resources.
We considered three sets of files, the first one with file size averaging $10 \mathrm{Mbytes}$, the second one with file size averaging $50 \mathrm{Mbytes}$, and the third one with file size averaging 100Mbytes.

Table 3 shows the average download time for direct client-server download without cooperation between neighboring devices.

This scenario is compared against other scenarios having more smart phones ( 2 to 4 ) downloading the same images from the server exploiting the proposed cooperative access architecture.

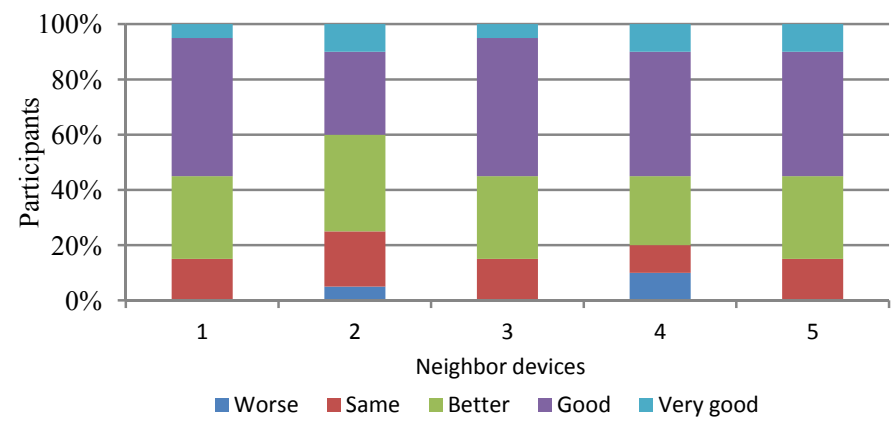

Fig. 5. Subjective evaluation of results.

Table 3: Average Downloading Time For Direct ClientSERVER DOWNLOAD

\begin{tabular}{cccc}
\hline \multirow{2}{*}{ File size } & \multicolumn{3}{c}{ Requests for single session } \\
\cline { 2 - 4 } & 1 & 10 & 100 \\
\hline 1 Mbyte & $7.4 \mathrm{sec}$ & $67 \mathrm{sec}$ & $712 \mathrm{sec}$ \\
\hline 5 Mbyte & $32 \mathrm{sec}$ & $295 \mathrm{sec}$ & $3127 \mathrm{sec}$ \\
\hline
\end{tabular}


Table 4: Average Downloading When 3 Neighbors Are COOPERATING

\begin{tabular}{cccc}
\hline \multirow{2}{*}{ File size } & \multicolumn{4}{c}{ Requests for single session } \\
\cline { 2 - 4 } & 1 & 10 & 100 \\
\hline 1 Mbyte & $3.5 \mathrm{sec}$ & $28 \mathrm{sec}$ & $351 \mathrm{sec}$ \\
\hline
\end{tabular}

The results obtained during the experimental test are shown in Table 4. This table reports the average downloading time for cooperative wireless access when 3 neighbors are cooperating. For example, for a hundred Mbytes the downloading time is $351 \mathrm{sec}$.

Fig. 4 compares the average download time for three scenarios: single user downloading without cooperation between neighboring users ( 1 user curve); two users cooperating in the downloading process; four users cooperating in the downloading process.

As shown in Fig. 4, for the 100Mbyte case there is a higher gain between the 2-user case and the 4-user case.

Nevertheless, downloading times are very different within one testing session and among one another. In general, experimental tests show that the cooperative download speeds up the downloading process.

The proposed architecture was then analyzed by subjective user experience measurement.

Thirty people were asked to download the files without network cooperation and then with network cooperation enabled, and to make a judgment between 1 and 5 ( $1=$ more time was required for image downloading, $2=$ no difference, $3=$ slightly faster, $4=$ faster, $5=$ very fast). The test was repeated for a number of neighboring devices going from 1 to 5 .

Fig. 5 summarizes the subjective evaluation results. In most of the cases the user experience has been improved. Users using the cooperative network were able to download image files faster than without the cooperation.

\section{CONCLUSIONS}

Network cooperation is a paradigm that can be exploited for reducing downloading time of several files or very large files from mobile devices. The main contributions of this paper are design and implementation of network cooperation between smartphone devices and empirical evaluation of the resulting performances. An algorithm for reducing the downloading time has been proposed. The proposed algorithm has allowed a reduction in WWAN network load and downloading time of multimedia contents from cellular networks. Comparisons have been made between direct download exploiting a single WWAN connection and cooperative download exploiting multiple WWAN connections and packet exchange across WLAN connections.

\section{REFERENCES}

[1] K. Yasumoto, Y. Takamatsu, W. Sun, M. Ito, "Energy-efficient cooperative download for smartphone users through contact time estimation," IEEE 8th International Conference on Wireless and Mobile Computing, Networking and Communications (WiMob), 2012.
[2] Y. Zou, J. Zhu, and R. Zhang, "Exploiting Network Cooperation in Green Wireless Communication," IEEE Transactions on Communications, vol. 61, no. 3, 2013.

[3] F.H.P. Fitzek, J. Heide, M.V. Pedersen, M. Katz, "Implementation of Network Coding for Social Mobile Clouds," IEEE Signal Processing Magazine, January 2013.

[4] C. Perra, D.D. Giusto, "An image browsing application based on JPEG XR," IEEE International Workshop on Content-Based Multimedia Indexing, 2008, 18-20 June 2008, pp. 396-401.

[5] B. Saba, C. Perra, D.D. Giusto, "Interactive image viewing in mobile devices based on JPEG XR," Mobile Multimedia Communications, Lecture notes of the institute for computer sciences, social informatics and telecommunications engineering, 2011.

[6] B. Saba, C. Perra, D.D. Giusto, "Image transmission over errorprone channels," IEEE 19th Telecommunications forum (TELFOR), 2011.

[7] C. Perra, "Re-encoding JPEG images for smart phone applications," IEEE 21st Telecommunications forum (TELFOR), 2013.

[8] C. Perra, P.A. Pes, D.D. Giusto, "High-Frequency Error Recovery In JPEG XR Coded Images," IEEE International Conference on Image Processing (ICIP), 2011.

[9] C. Perra, "Wireless cooperative access to images," The 9th IEEE International Wireless Communications \& Mobile Computing Conference (IWCMC), 2013

[10] M. Fadda, M. Murroni, C. Perra C, V. Popescu, "TV White Spaces Exploitation for Multimedia Signal Distribution," Signal Processing-Image Communication, 2012.

[11] S.K. Dandapat, R. Niranjan, N. Ganguly, "Framework for Collaborative Download in WirelessMobile Environment," 13th International Conference on Mobile Data Management, 2012.

[12] B. Xing, K. Seada, N. Venkatasubramanian, "Proximiter: Enabling mobile proximity-based content sharing on portable devices," IEEE International Conference on Pervasive Computing and Communications, 2009.

[13] R. Ahlswede, Ning Cai, S.-Y. R. Li, and R. W. Yeung, "Network information flow," in Proc. IEEE Trans. Inform. Theory, pp. 1204 1216,2000

[14] S. Katti, H. Rahul, W. Hu, D. Katabi, M. Medard, and J. Crowcroft, "XORs in the air: Practical wireless network coding," in Proc. Conf. Applications Technol. Architectures Protocols Comput. Commun., pp. 243-254, 2006.

[15] M. V. Pedersen, F. H. P. Fitzek, and T. Larsen, "Implementation and performance evaluation of network coding for cooperative mobile devices," in Proc. IEEE Cognitive Cooperative Wireless Networks Workshop, pp. 91-96, 2008.

[16] X. Liu, G. Cheung, C.-N. Chuah, "Structured network coding and cooperative wireless ad-hoc peer-to-peer repair for WWAN video broadcast," IEEE Trans. Multimedia, vol. 11, no. 4, pp. 730-741, June 2009

[17] G. Ananthanarayanan, V.N. Padmanabhan, L. Ravindranath, C. A. Thekkath, "COMBINE: leveraging the power of wireless peers through collaborative downloading," In Proceedings of the 5th international conference on Mobile systems, applications and services, MobiSys 07, pages 286298, New York, NY, USA, 2007.

[18] L. Keller, A. Le, B. Cici, H. Seferoglu, C. Fragouli, "A. Markopoulou MicroCast: Cooperative Video Streaming on Smartphones," Proceedings of the 10th international conference on Mobile systems, applications, and services, 2012.

[19] J. Heide, F.H.P. Fitzek, M.V. Pedersen, M. Katz, Marcos, "Green mobile clouds: Network coding and user cooperation for improved energy efficiency," IEEE 1st International Conference on Cloud Networking (CLOUDNET), 2012.

[20] EX20 - M. Pedersen, F. Fitzek, and T. Larsen, "Implementation and Performance Evaluation of Network Coding for Cooperative Mobile Devices," in IEEE International Conference on Communications, May 2008.

[21] Y. Wu, Z. Zhang, C. Wu, Z. Li, F.C.M. Lau, "CloudMoV: CloudBased Mobile Social TV," IEEE Transactions on Multimedia, Vol. 15, No. 4, June 2013.

[22] C. Perra, "Wireless Cooperative Access for Next Generation Mobility Applications," IEEE 21st Telecommunications forum (TELFOR), 2013. 